\title{
Is "nonusual interstitial pneumonia" an acceptable diagnosis?
}

\author{
J. Behr
}

The recently published "Classification of Idiopathic Interstitial Pneumonias (IIPs)" differentiates seven disease entities [1] as follows: 1) usual interstitial pneuminia (UIP), 2) desquamative interstitial pneumonia (DIP), 3) respiratory bronchiolitis-associated interstitial lung disease (RBILD), 4) nonspecific interstitial pneumonia (NSIP), 5) acute interstitial pneumonia (AIP), 6) cryptogenic organising pneumonia (COP), and 7) lymphoid interstitial pneumonia (LIP). These entities have primarily been defined pathologically $[1,2]$. However, in clinical practice only a minority of patients with suspected IIP undergo surgical lung biopsy [3]. Diagnostic criteria that allow a clinical diagnosis in the absence of a surgical lung biopsy have been defined only for UIP [4]. In two previous publications the overall accuracy of a clinical diagnosis of UIP varied from $62-77 \%$ and the overall accuracy of a radiological diagnosis of UIP was about $75 \%[5,6]$. The most important noninvasive diagnostic tool for the diagnosis and differentiation of IIPs is the high-resolution computed tomography (HRCT) [7, 8 , 9]. It has also been demonstrated that a HRCT showing features not typical of UIP is associated with a relatively good prognosis, which is comparable to that of histologically proven NSIP [10]. The presence of a significant amount of ground glass opacities and the absence of honeycombing is especially indicative of a favourable response to corticosteroids and a good prognosis in general. However, the discussion on how to diagnose the different forms of IIPs is still ongoing, despite the general contention that a surgical lung biopsy is the "gold standard".

In this issue of the European Respiratory Journal, WATANABE et al. [11] present a study which investigated the usefulness of transbronchial lung biopsy (TBLB) to predict the response to steroids in patients with IIPs who were unlikely to be UIP, but were not surgically biopsied. They report that selected patients, who showed a dense cellular interstitial infiltration on TBLB, experienced a similar good response to steroid treatment as patients with NSIP proven by surgical biopsy. The study, however, has several limitations, especially due to the small number of patients (only nine such "non-UIP" patients were evaluated) and the

Dept of Internal Medicine I, Division for Pulmonary Diseases, Klinikum Grosshadern, University of Munich, Munich, Germany.

Correspondence: J. Behr, Dept of Internal Medicine I, Division for Pulmonary Diseases, Klinikum Grosshadern, University of Munich, Marchioninistr. 15, 81377 Munich, Germany. Fax: 89 70958877. E-mail: jbehr@med1.med.uni-muenchen.de retrospective design. Most importantly, the patients in whom the TBLB was employed as an additional diagnostic test, had been preselected on the basis of HRCTs showing a "non-UIP" pattern in all of them [11]. DANIIL et al. [10] have previously reported that a high proportion of these patients do respond to antiinflammatory therapy and have a relatively good prognosis, comparable to patients with histologically proven NSIP. The additional prognostic contribution of a histological finding of "dense mononuclear cell infiltration in thickened alveolar septa" in a TBLB specimen from IIP-patients can not be assessed from the data presented by WATANABE et al. [11]. This is especially true because there is no proper control group in their paper. The comparison of the "nonUIP" group with a group of 10 patients with histologically proven NSIP is suggestive but does not prove that TBLB is necessary in those patients, in whom a favourable prognosis can be expected from the HRCT findings alone. In order to demonstrate an additional diagnostic impact of TBLB over HRCT alone, two control groups would be necessary: one composed of patients with HRCT findings not compatible with UIP who would not undergo any type of lung biopsy and one of patients with HRCT findings not compatible with UIP who would undergo TBLB and would not show a "dense mononuclear cell infiltration in thickened alveolar septa" on this type of biopsy.

Moreover, it should be noted that it is still not possible to identify different forms of IIPs with the help of TBLB or to make a specific diagnostic contribution with TBLB beyond the exclusion of granulomatous lung diseases, malignancy and some infections. A general strategy of using HRCT and TBLB for diagnosing IIPs would introduce an unacceptable simplification into the differential diagnosis of the IIPs, resulting in only two possible diagnoses, "UIP" and "non-UIP". The progress in the last few years in the field of IIPs, which has allowed clinical trials in larger homogeneous patient populations to be performed for the first time, would be challenged if these strategies became general clinical practice.

Nevertheless, WATANABE et al. [11] have contributed to an ongoing discussion. Their paper may inspire other groups to investigate more thoroughly the diagnostic yield of transbronchial lung biopsy in idiopathic interstitial pneumonias with a clinical pattern not compatible with usual interstitial pneuminia. But until the results of such studies are available, 
including adequate control groups, surgical lung biopsies should be considered as the gold standard for diagnosing idiopathic interstitial pneumonias, except for typical cases of usual interstitial pneuminia fulfilling the established clinical and high-resolution computed tomography criteria [4].

\section{References}

1. American Thoracic Society/European Respiratory Society International Multidisciplinary Consensus Classification of the Idiopathic Interstitial Pneumonias. Am J Respir Crit Care Med 2002; 165: 277-304.

2. Katzenstein A-L, Myers JL. Idiopathic pulmonary fibrosis. Clinical relevance of pathological calssification. Am J Respir Crit Care Med 1998; 157: 13011315.

3. Johnston ID, Gomm SA, Kalra S, Woodcock AA, Evans CC, Hind CR. The management of cryptogenic fibrosing alveolitis in three regions of the United Kindom. Eur Respir J 1993; 6: 891-893.

4. ATS/ERS Interational Consensus Statement. Idiopathic pulmonary fibrosis: diagnosis and treatment. Am J Respir Crit Car Med 2000; 161: 646-664.

5. Raghu G, Mageto YN, Lockhart D, Schmidt RA, Wood DE, Godwin JD. The accuracy of the clinical diagnosis of new-onset idiopathic pulmonary fibrosis and other interstitial lung diseases: a prospective study. Chest 1999; 116: 1168-1174.

6. Hunninghake GW, Zimmermann MB, Schwartz DA, et al. Utility of a lung biopsy for the diagnosis of idiopathic pulmonary fibrosis. Am J Respir Crit Care Med 2001; 164: 193-196.

7. Müller NL, Miller RR, Webb WR, Evans KG, Ostrow DN. Fibrosing alveolitis: CT-pathologic correlations. Radiology 1986; 160: 585-588.

8. Bergin CJ, Müler NL. CT of interstitial lung disease: a diagnostic approach. Am J Roentgenol 1987; 148: 915.

9. Tung KT, Wells AU, Rubens MB, Kirk JM, du Bois RM, Hansell DM. Accuracy of the typical computed tomographic appearances of fibrosing alveolitis. Thorax 1993; 48: 334-338.

10. Daniil ZD, Gilchrist FC, Nicholson AG, et al. A histologic pattern of nonspecific interstitial pneumonia is associated with a better prognosis than usual interstitial pneumonia in patients with cryptogenic fibrosing alveolitis. Am J Respir Crit Care Med 1999; 160: 899-905.

11. Watanabe K, Higuchi K, Ninomiya K, et al. Steroid treatment based on the findings of transbronchial biopsy in idiopathic interstitial pneumonia. Eur Respir $J$ 2002; 20: 1213-1219. 